\title{
Controlled Fusion: Magnetic and Inertial, Promises and Pitfalls
}

\author{
Kenell James Touryan \\ College of Science and Engineering, American University of Armenia, Yerevan, Armenia
}

\section{Email address:}

kenell@comcast.net

\section{To cite this article:}

Kenell James Touryan. Controlled Fusion: Magnetic and Inertial, Promises and Pitfalls. American Journal of Electrical Power and Energy Systems. Vol. 9, No. 6, 2020, pp. 104-108. doi: 10.11648/j.epes.20200906.12

Received: November 30, 2020; Accepted: December 14, 2020; Published: December 22, 2020

\begin{abstract}
As with biomass, hydro, solar and wind power, fusion power can also generate clean energy, using deuterium, an isotope of hydrogen, abundantly available in our oceans. Our sun uses hydrogen in a fusion process to generate power. It has been demonstrated that fusion power can be generated on earth, under carefully controlled conditions using deuterium and tritium instead of hydrogen. There are two fundamental approaches to controlled fusion: magnetic confinement fusion (MCF) first proposed at Princeton University in 1951, and inertial confinement fusion (ICF) that followed shortly thereafter, first proposed at the Lawrence Livermore Laboratories in 1970. Progress made on magnetic fusion led to the planning and construction of ITER (International Thermonuclear Experimental Reactor), expected to be completed in 2035. In this article, we explain the processes necessary to generate fusion power through MCF and ICF. Unlike nuclear power, as a practical means to generate electricity, controlled fusion has presented the technical/scientific community with a plethora of very difficult challenges. It is only recently, after decades of intense research in many laboratories worldwide, that we have begun to see devices being built on a fusion reactor scale and hence the design of ITER. The challenges are many but require patience and perseverance.
\end{abstract}

Keywords: Magnetic Fusion, Inertial Fusion, Controlled Fusion, Plasma Dynamics, ITER, Plasma Confinement, Clean Energy

\section{Introduction}

How does one re-create the fusion power source that energizes our giant sun in a manmade laboratory? In Genesis 1 we read that on the fourth day... God made two great lights... the greater light [the Sun] to govern the day..."

Our sun is the major source of energy and light that supports life on Planet Earth. We know now that the source of energy from the sun is fusion power, generated when hydrogen ions (and other low atomic weight elements to be discussed below) fuse together under immense pressures and multi-million degree temperatures, converting mass into energy according to Einstein's simple equation $\mathrm{E}=\mathrm{mc}^{2}$ (where $\mathrm{m}$ is the mass and $\mathrm{c}$ is the speed of light). What is even more mind-boggling, the same source of fusion energy powers the myriad stars $\left(10^{24}\right)$ that make up the $10^{12}$ galaxies in our universe.

Clean and renewable energy, with a near boundless fuel source (hydrogen and its isotopes) has been a huge incentive to attempt the Herculean task of generating power through fusion. It was Lyman Spitzer [1] of Princeton who proposed in 1951 a magnetically confined, high temperature plasma in a device called the Stellarator for fusion. A system which is often considered impossible, but a challenge before us, which if solved, and used for peaceful purposes, could be a great contribution toward a clean energy planet. The same year, Andrei Sakharov and Igor Tamm of the Soviet Union proposed a similar magnetically confined fusion device called a Tokamak [2]. The fuel used in these devices is heavy hydrogen, called deuterium, which is abundant in sea water (details below).

There are two fundamental approaches to controlled fusion [3]: magnetically confined, low pressure systems and inertial confinement systems where lasers and other techniques are used to achieve high pressures. The term controlled is used to distinguish it from the fusion technology used explosively in thermonuclear weapons.

Unlike traditional fossil fuel sources, other clean energy systems like solar cells, wind, biomass energy sources and even nuclear power, fusion power in the laboratory has frustrated scientists and engineers at every turn in its development, raising unforeseen technical difficulties. They 
occur when confining and heating the fuel at very high temperatures (called a plasma) for durations long enough to demonstrate the generation of steady power, which is on the order of 400 to 600 seconds for magnetically confined systems (see Figure 1a or 1b). This has been the experience for all the major players like the European Union, Russia (where fusion research began during USSR era), the US, Japan and more recently, China, South Korea, and India.

When I graduated from Princeton in 1962, I was invited to join the Stellarator team with the vision of having a prototype device ready for commercialization in the 1980 time-frame! Sad to say, it is now 2020 and the multiple research fusion experiments worldwide have not made it possible to design and build a commercial power plant, let alone prototypes.

\section{Magnetic Confinement Fusion (MCF)}

As mentioned in the introduction, the thermonuclear reactions that fuel the sun (and in all stars) begins with the hydrogen ions, i.e., protons fusing together under immense pressure and multimillion-degree temperatures. Those conditions cannot be duplicated on earth. The next best approach is to use the heavy isotopes of hydrogen, deuterium and tritium, that have large enough of a cross section, or probability to allow fusion to occur, at more 'moderate' temperatures and pressures, with the anticipation that fusion can be attained in laboratories and/or commercial fusion power plants on earth.

The first generation of fusion reactors will rely on the following reactions:

$$
\begin{gathered}
\mathrm{D}+\mathrm{T}->\mathrm{He}(3.5 \mathrm{MeV})+\mathrm{n}(14.1 \mathrm{MeV}) \\
\mathrm{n}+{ }^{6} \mathrm{Li}->\mathrm{He}(2.1 \mathrm{MeV})+\mathrm{T}(2.47 \mathrm{MeV})
\end{gathered}
$$

Here $\mathrm{n}$ is neutron, $\mathrm{D}$ and $\mathrm{T}$ are isotopes of hydrogen with $\mathrm{D}$ having one proton and one neutron and $\mathrm{T}$, one proton and two neutrons. He is helium and ${ }^{6} \mathrm{Li}$ is an isotope of lithium. Because $\mathrm{Li}$ is relatively rare, the fusion power will be primarily from the $\mathrm{D}-\mathrm{T}$ reaction. $\mathrm{MeV}$ stands for million electron volt energy $(1 \mathrm{eV}$ is equivalent to 11,606 degrees Celsius).

The D-T reaction has the lowest ignition temperature and the lowest confinement requirement of all other reactions. However, most of the energy comes out in the form of 14$\mathrm{MeV}$ neutrons, and converted into a usable form in a total heat cycle whose thermodynamic efficiency is $40 \%$. Furthermore, neutrons cause damage to the reactor walls (made from various carbides, tungsten and/or carbon fiber components), making them not only radioactive, but losing structural integrity so they have to be replaced and disposed of periodically. The other disadvantage is that unlike deuterium, which is abundant in sea water, tritium does not occur in nature, is radioactive (with a 12-year half-life) and has to be bred through Equation 2 above. Lithium is abundant in the earth's crust, but its ${ }^{6} \mathrm{Li}$ isotope comprises only $7.5 \%$ of the total amount of Lithium. All this indicates that controlled fusion, although dependent on abundant fuel like Deuterium, is only partially clean.

There are more benign ways of producing fusion power, by only using the Deuterium-Deuterium reaction without breeding Tritium and only $34 \%$ of the energy appearing as neutrons. However, the temperatures required are much higher for the D-D reaction. Finally, if very high temperatures can be attained for ignition, there is a third set of reactions that involve $\mathrm{D}, \mathrm{He}, \mathrm{Li}$ and $\mathrm{Be}$ (beryllium) that can generate only charged particles, but no neutrons, thus reducing wall damage and radioactivity.

\subsection{Necessity for Plasmas}

High temperatures are essential for fusion to occur, ergo the need of ionized gasses called a plasma. Since ions are positively charged, the Coulomb force of repulsion must be overcome before the above reactions can occur. For instance, the cross section for the D-T reaction rises sharply at milliondegree temperatures. The solution is to generate a plasma with a mean energy that corresponds to these temperatures. Such a collection of plasma particles is called a Maxwellian distribution, and there should be enough energetic particles on the high end of the distribution that could collide and fuse.

To produce more energy from net fusion power, it is required to heat the plasma and reduce the radiation losses. Conditions should be met on the plasma density " $n$ " and confinement time $\tau$, as well as on the temperature T. This leads to what is known as the Lawson Criterion $\mathrm{nT} \tau$ [4], which states that the you must hold together enough particles (n) at a high enough temperature ( $\mathrm{T}$ ) for a confinement time long enough $(\tau)$ for fusion to occur. The minimum value for $\mathrm{nT} \tau$ should be $10^{14} \mathrm{~cm}^{-3}$ sec for D-T reactions at about 25 $\mathrm{KeV}$ or million degrees Celsius, and $10^{16}$ for D-D reactions at about $50 \mathrm{KeV}$ or 2 million degrees Celsius. The minimum confinement time should be $\sim 0.1 \mathrm{sec}$.

Figures 1 and 2 show sketches of a Stellarator and a Tokamak device with the magnetic fields confining the plasma away from the walls of the devices. The difference between the two types of confinement are shown in the subtitles. The Stellarator requires complicated magnetic field coils. They are simpler in Tokamaks, but the confining magnetic field can go away if an instability develops in the plasma (see below). This is why both approaches continue to be studied.

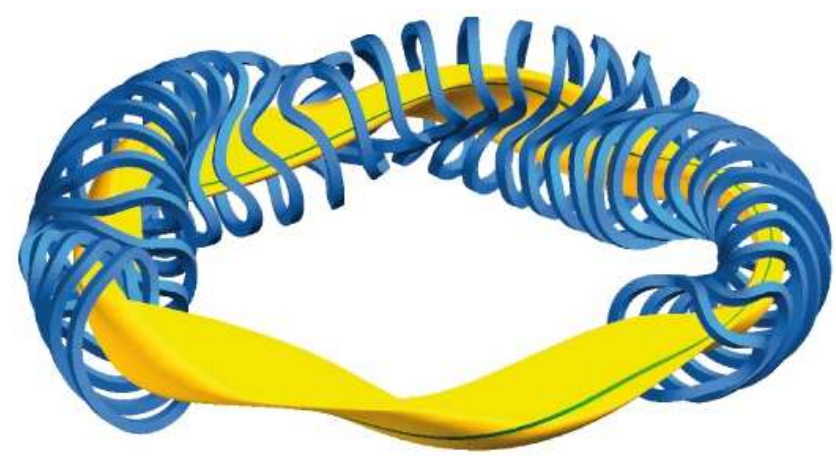

Figure 1. Illustration of a Stellarator showing the complex magnetic field coils (blue) for creating the twisted magnetic fields that are needed to confine the plasma (yellow). Without such a geometry, the magnetic field would not be able to keep the plasma particles from drifting out of the machine. https://www.ipp.mpg.de/w7x. 
One of the major challenges of these devices is the interaction of the magnetic field and the hot plasma. The plasma acts like a doughnut-shaped fluid, and instabilities can form that look like a "kink" that can push the plasma to the walls before the plasma has the required confinement time - at least 0.1 seconds for the D-T reaction - to initiate fusion. There are other instabilities that act to quench the plasma and shut down the fusion process. Without going into details, there are three ways to prevent instabilities: creating "shear" in the plasma flow to provide a barrier against plasma movement, creating a deeper "well" in the magnetic confining field, and adding magnetic fields to "push" the plasma back in place $[5,6]$. The Stellarator and Tokamak machines use different magnetic field configurations to deal with these instabilities, in an attempt to increase the time the plasma has to remain stable at temperatures to allow practical demonstration of fusion (e.g., 400 to 600 seconds).

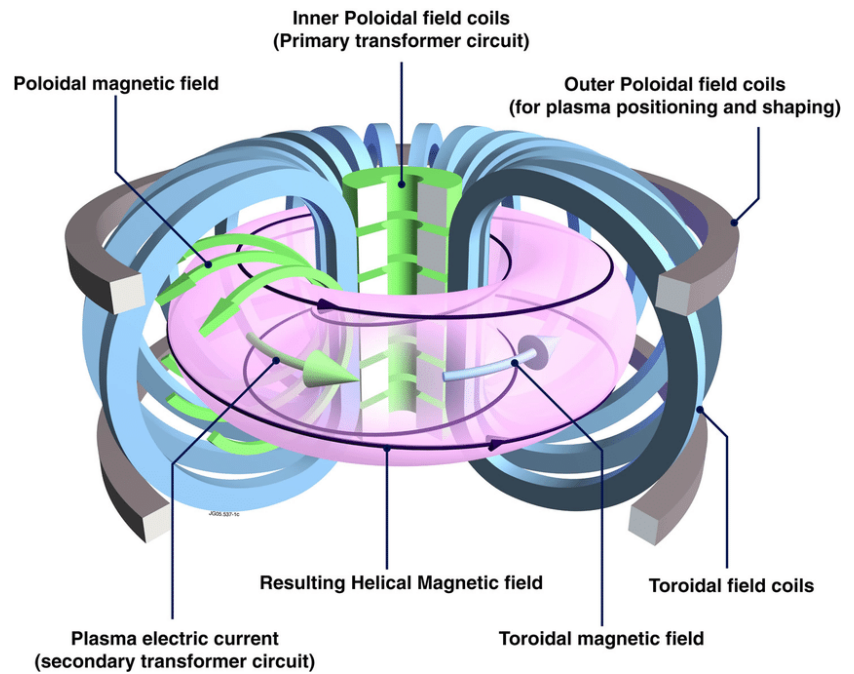

Figure 2. Illustration of a Tokomak. The toroidal field (blue) coils create a magnetic field that goes around the torus (toroidal magnetic field). The plasma itself is an electrical conductor, so a coil in the center of the tokamak can induce a plasma electric current to flow through it as in a transformer. The result is a poloidal magnetic field (green arrows). The combination of the toroidal and poloidal magnetic fields creates the helical magnetic field that is required to contain the plasma. https://www.ipp.mpg.de/w7x.

\subsection{Prospects for Commercial Fusion Using Magnetically Confined Plasmas}

Progress made on magnetic fusion has led to the planning and construction of ITER ('The Way' in Latin, see Figure 3), a thermonuclear reactor scale fusion research facility in Provence, France, funded by the major fusion players. The facility will cost $\$ 25 \mathrm{~B}$ and go operational in 2035. It will provide the opportunity to study plasmas where it is expected that the total heating will come from fusion reactions that could produce $500 \mathrm{MW}$ of power for 400 to 600 seconds (Figure 3). Estimates including 50MW power needed as input heating to power the plasma, plus utility related efficiency considerations, may seriously degrade the planned $500 \mathrm{MW}$ fusion power output to a level $(150 \mathrm{MW})$ which is not sufficient to demonstrate practical commercial use. For
ITER to perform as best as possible, it will require continuous input from parallel activities in various laboratory devices, to keep dealing with scientific and technological issues while ITER is in construction (see section above).

It should be noted that significant fusion power has been achieved in magnetically confined plasmas in laboratories, up to $10 \mathrm{MW}$ in Tokamak Fusion Test Reactor in the US and up to $16 \mathrm{MW}$ in the Joint European Torus in the UK. Examples of ongoing improvements include: 1-Tokamaks in China (EAST) and South Korea (KSTAR) with superconducting magnets to enable long plasma durations; 2-A new stellarator in Germany (Wendelstein 7-X) with "modular" superconducting magnetic field coils as you would need to build a reactor; 3-Compact tokamaks in the US (NSTX-U) and the UK (MAST-U) to confine plasmas more efficiently with lower magnetic fields [7].

A recent announcement from MIT, describes a renovated Tokamak called SPARC, that uses high temperature superconducting tapes coated with a compound called yttriumbarium-copper oxide (YBCO) as magnets. It is estimated to reduce the size of the device and make it more cost effective $[8,9]$.

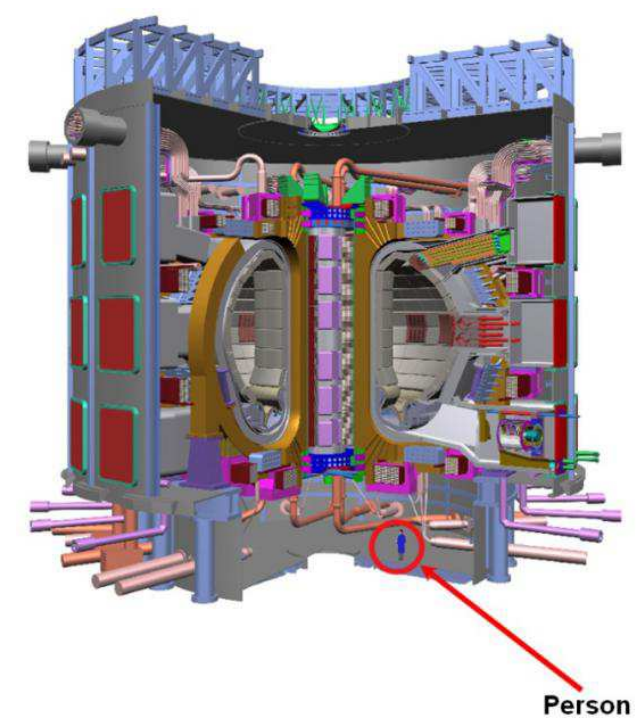

Figure 3. Schematic of ITER, a thermonuclear reactor-scale fusion device under construction in southern France. The massive scale of the project is illustrated by the person near the base of the machine. http://www.ipp.mpg.de/.

Following the Manheimer estimates (see section 2.2) [10], the question becomes, is it really worth spending billions on ITER if useful power may not be available in this century? There is no question that fusion as a clean source of energy, with abundant supply of fuel like deuterium, is an essential component for providing the increasing energy needs of the world. The question today is one of cost effectiveness. Spending huge sums of money on fusion at this stage of development may not be an immediate priority, given the many other pressing priorities required to meet basic human needs. It would be wise to continue research on developing fusion power, but cognizant of our call to good stewardship, at a pace that also recognizes the need to address humanity's near-term concerns. 


\section{Inertial Confinement Fusion (ICF)}

A different fusion system called the ICF started at the Lawrence Livermore National Laboratory (LLNL) of the US Department of Energy (USDOE) in 1970. It uses high energy lasers to focus on a small spherical pellet, or capsule filled with a mix of D-T. Energy from a bank of lasers (see below) heats the surface of the pellet, in a tiny fraction of a second, into a plasma which explodes off the surface of the capsule. The remaining portion of the target is driven inward, eventually compressing it into a small point of extremely high density $\left(10^{26} \mathrm{~cm}^{-3}\right)$. The rapid blow off also creates a shock wave that travels toward the center of the compressed fuel from all sides. When it reaches the center of the fuel, a small volume is further heated and compressed to a greater degree (Figure 4). When the temperature and density of that small spot are raised high enough, fusion reactions occur and release energy [11].

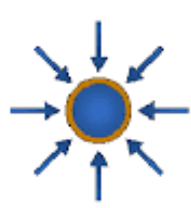

a)

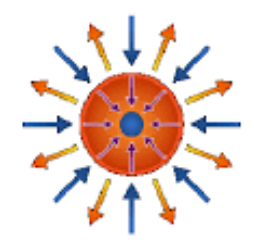

b)

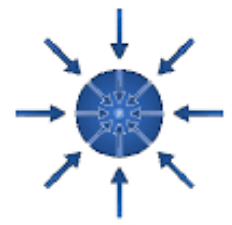

c)

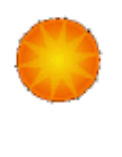

d)
Figure 4. Sketch of the imploding capsule in the inertial confinement system. Blue arrows illustrate the incoming laser beams focused on the capsule (a), followed by the explosion of the capsule surface (b) and the implosion that compresses the capsule core (c) and creates a fusion plasma (d). https://en.wikipedia.org/wiki/Inertial_confinement_fusion.

The fusion reactions release high-energy ionized particles, some of which, primarily alpha particles (i. e., ionized Helium), collide with the surrounding high-density fuel and heat it further. If this process deposits enough energy in a given area, it can cause that fuel to undergo fusion as well. However, the fuel is also losing heat through x-ray losses and hot electrons leaving the fuel area, so the rate of alpha heating must be greater than these losses, a condition known as bootstrapping. Given the right overall conditions of the compressed fuel-high enough density and temperaturethis bootstrapping process will result in a chain reaction burning outward from the center where the shock wave started the reaction. This is a condition known as ignition, which will lead to a significant portion of the fuel in the target undergoing fusion and releasing large amounts of energy [12].

Most ICF experiments that started in 2009, at a cost of $\$ 3.5$ billion, have used lasers to heat the target. Experiments on the world's most powerful laser started in 2009. The first laser was demonstrated in 1960 and by early 1970's high power lasers were beginning to be made commercially. Calculations show that the energy must be delivered quickly $\left(10^{-11}\right.$ seconds) in order to compress the core before it disassembles. The laser energy also must be focused extremely evenly across the target's outer surface in order to collapse the fuel into a symmetric core. Although other drivers have been suggested, notably heavy ions driven in particle accelerators, the solid state laser driven ICF systems are currently the most efficient.

\subsection{Driver Laser System}

The heart of the ICF system at LLNL is the 192-beam neodymium lasers and their amplifiers, that are switched on simultaneously in picoseconds, to deliver a multi-megawatt punch to the tiny capsule, a millimeter across and filled 5050 with D-T. If all goes well, the implosion will compress the fuel into a spherical "blob," 20 times the density of lead and some 30 micrometers across, in which the very center can reach 100 million degrees Kelvin. Brute force, e.g., the application of 1 mega joule alone, is not enough to create fusion. Ignition requires getting the laser to heat the capsule evenly from all directions; a difficult finesse that was resolved only recently [13].

In 1994, ICF at LLNL was christened the National Ignition Facility (NIF). The ground for the NIF was broken in 1997 on a LLNL site. Following a period of several years on 14 June 2018 researchers at LLNL doubled previous records both for neutron yield $\left(1.9 \times 10^{16}\right)$ and fusion energy output, now at 54 kilojoules from a diamond capsule containing cryogenic D-T fuel. It was accomplished by maintaining the symmetry by changing both the capsules and the cylinders in which the capsules are suspended. This has been called a critical step towards in the quest in achieving laboratory ignition. "However, as part of the National Nuclear Security Administration (NNSA) of the USDOE, the primary justification for NIF is its relevance to nuclear weapons physics, mimicking on a laboratory scale the dynamics in a weapon's fusion stages. Laser fusion as future energy source is thus the secondary mission for NIF!"

\subsection{Research at Other Laboratories}

Researchers at Sandia National Laboratories in Albuquerque, NM have been developing another technique that relies on a phenomenon called the pinch effect. Sandia has a huge device called the Z-machine, which stores enormous amounts of electrical energy and then produces intense current pulses that can generate huge magnetic fields [14]. These fields compress a metal cylinder filled with D-T and may be the basis for a fusion device. Finally, there is one more system being used today called 'indirect drive' where laser energy hits a hohlraum which in turn generates a bath of $\mathrm{x}$-rays that irradiate the capsule more uniformly from all directions.

Other labs working on the ICF principle are Los Alamos National Laboratory, the Laboratory for Laser Energetics (LLE) in Rochester, NY and the Naval Research Laboratory (NRL) in Washington, DC in the US, and the Laser Mégajoule in Bordeaux, France. In spite of the above successes, the Lawson criterion for generating fusion power has yet to be achieved. The USDOE changed the direction of NIF by stating that barring an unforeseen technical breakthrough and given today's configuration of the NIF 
system, achieving ignition in the near term (one to two years) is unlikely, and is uncertain in the mid-term (five years). The question to NNSA has come down to one of "if NIF reaching ignition in its current configuration [mentioned above], and not when it will occur: It is therefore recommended to make better use of the NIF facilities, and those not designed to achieve ignition, to better understand the underlying physics of the compressed fuel, known as high-energy density plasma." [15]

\subsection{A Personal Observation}

In 1978, during one of my visits to LLNL after the lab had just started their proof of concept tests with NOVA, a 10beam laser, I stepped out of the gigantic, multibillion dollar ICF facility, with all its complex man-made paraphernalia in its attempt to create fusion in a millimeter-sized capsule, and my eyes caught our setting sun, the giant fusion machine set in place by the Creator. It has existed for many millions of years and has enough fuel for many millions more, providing uninterrupted energy to our planet. Suddenly I realized that it is far more practical and economical to capture that energy in solar cells instead. At that instant, I had a sudden ' $a h a^{\prime}$ moment that led me to devote the rest of my life to developing renewable energy technologies.

However, efforts to harness fusion energy for peaceful purposes should be pursued. It is a sad commentary to realize that thermonuclear power was first used to develop weapons of mass destruction. On the other hand, even with the largescale introduction of solar and wind technologies, it is still important to have base power plants run on clean energy, such as fusion power, to smooth over the intermittency issues of solar and wind energy sources.

\section{Conclusion}

Being good stewards of our planet, we should encourage proper use of technology for the benefit of all life.. Controlled fusion, both magnetic (MCF) and inertial (ICF) are such technologies. Controlled fusion, which in the past was considered impossible, continues to be a challenge for scientists but if solved and used for peaceful purposes, will offer a great contribution toward a clean energy planet. It should be noted that magnetic fusion is proceeding on schedule, with ITER being the next large-scale goal. Inertial fusion however, has been re-directed toward laboratory scale demonstrations of nuclear weapons testing, in its various stages. Laser fusion for energy sources has thus become a secondary mission for NIF.

It is worthwhile to recall the statement God made regarding humans and their capability to deal with extremely demanding challenges. Observing the Tower of Babel, God said, "...nothing they plan to do will be impossible for them" (Genesis 11:6).

\section{References}

[1] Spitzer, L., Physics of Fluids 1, 253 (1958).

[2] Wesson, J., Tokamaks, 2011 (Oxford: Oxford University Press).

[3] Chen, Francis F., Introduction to Plasma Physics, First Edition, 1974 (Plenum Press NY), Chapter 9.

[4] Lawson, J. D., Proceedings of the Physical Society, Section B. 70, 6 (1957).

[5] Hawryluk, Richard, Rev. Mod. Physics 70, 537 (1998).

[6] Chen, Francis F., Introduction to Plasma Physics, Third Edition, 2016 (Berlin: Springer) Chapter 10 for description of other confinement approaches.

[7] Y-KM Peng et al "Component Test Facility Based on Spherical Tokamak", Plasma Physics and Controlled Fusion, $2005 \mathrm{Vol} 47,12 \mathrm{~B}$.

[8] Greenwalk, Martin, Status of the SPARC physics basis, Journal of Plasma Physics, 2020; 86 (5) DOI: https://doi.org/10.1017S0022377820001063.

[9] Creely, A. J. et al Overview of the SPARC Tokamak, Jof Plasma Physics 86 (5), 2020.

[10] Manheimer, W., Physics Today 73, 7, 10 (2020).

[11] Lindl, J. D., Phys. Plasmas 2, 3933 (1995).

[12] LePape, S., et al., Phys. Rev. Letter 120, 245003 (2018).

[13] Kramer, D., Physics Today, Politics and Policy, 17 June 2016.

[14] Private Communication, Dr Marshall M. Sluyter, Former Director of NIF, US Department of Energy, August, 2020.

[15] Clary, Daniel. 'Laser Fusion reactor approaches burning plasma milestone' Science, Nov 27, 2020. 